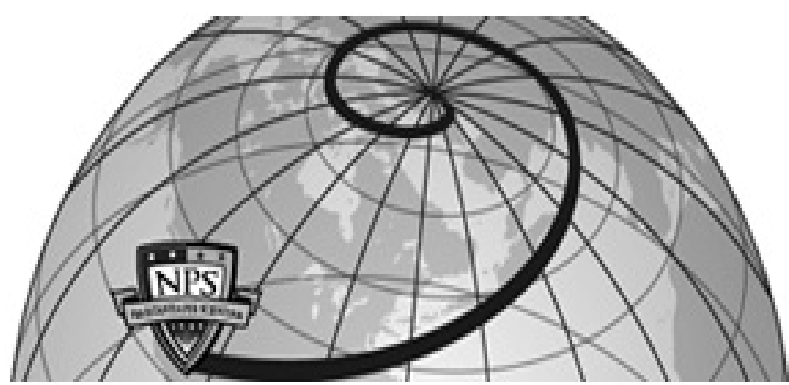

Calhoun: The NPS Institutional Archive DSpace Repository

High-Order Non-Reflecting Boundary

Conditions for the Linearized 2-D Euler Equations: No Mean Flow Case

Dea, John R.; Giraldo, Francis X.; Neta, Beny

https://hdl.handle.net/10945/40896

This publication is a work of the U.S. Government as defined in Title 17, United States Code, Section 101. Copyright protection is not available for this work in the United States.

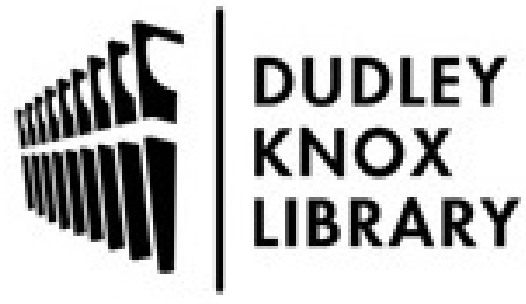

http://www.nps.edu/library
Calhoun is the Naval Postgraduate School's public access digital repository for research materials and institutional publications created by the NPS community. Calhoun is named for Professor of Mathematics Guy K. Calhoun, NPS's first appointed -- and published -- scholarly author.

Dudley Knox Library / Naval Postgraduate School 411 Dyer Road / 1 University Circle Monterey, California USA 93943 


\title{
High-Order Non-Reflecting Boundary Conditions for the Linearized 2-D Euler Equations: No Mean Flow Case
}

\author{
John R. Dea, Francis X. Giraldo, Beny Neta* \\ Department of Applied Mathematics \\ Naval Postgraduate School
}

July 23,2008

\begin{abstract}
Higdon-type non-reflecting boundary conditions (NRBCs) are developed for the 2-D linearized Euler equations with Coriolis forces. This implementation is applied to a simplified form of the equations, with the NRBCs applied to all four sides of the domain. We demonstrate the validity of the NRBCs to high order. We close with a list of areas for further research.
\end{abstract}

\section{Introduction}

To perform mesoscale atmospheric modeling on a computer, one immediately runs into the problem of defining the computational domain. At some point, there has to be an edge to the computational domain, but the physical atmosphere lacks any edges. How, then, can we define a computational boundary where no physical boundary exists? The answer of course is to define a non-reflecting boundary condition (NRBC). How best to define such a boundary has been an active area of research for approximately 30 years. Ideally, an NRBC will be stable, accurate, fast, and easy to implement; realistically, one must generally choose two or three of those criteria, at best.

There are typically two approaches to NRBC development. The first is to prescribe the behavior at the boundaries in such a way as to reduce any spurious reflections. Early examples include the Sommerfeld-conditionbased work of Orlanski [26] and the Padé approximations of Engquist and Majda [4, 5]. This approach was expanded by Higdon [13]-[19] and subsequently automated by Givoli, Neta, and van Joolen [7]-[10], [29]-[32]. The Orlanski scheme and the Engquist-Majda scheme are less accurate

${ }^{*}$ Corresponding author: Tel.: +1-831-656-2235; fax: +1-831-656-2355.

E-mail address: byneta@gmail.com (B. Neta) 
than their successors; however, the Higdon scheme and its offshoots suffer from very high computational overhead.

The second approach is to surround the domain with a more dispersive computational medium, so that incoming waves enter the absorbing layer and diffuse to zero before their reflections re-enter the original domain. Examples include the Perfectly Matched Layer (PML) developed by Bérenger [1], applied to the linearized shallow water equations by Navon et. al. [24] and to the linearized Euler equations by $\mathrm{Hu}$ [20, 21, 22], and the sponge layer used by Giraldo and Restelli [6]. This approach requires additional storage and computation time for the expanded domain, and some reflections are still evident when the theoretically-exact absorbing layer is applied to a discrete computational domain. Furthermore, the absorbing layer surrounding the computational medium precludes the possibility of incoming waves in a nested modeling environment; the incoming waves will be diffused to zero before they enter the computational domain.

Here we apply the Higdon scheme to the linearized Euler equations. We take advantage of the Givoli-Neta-van Joolen automation and make subsequent improvements to reduce the computational overhead. This method removes approximately $97 \%$ of the Sommerfeld condition's reflection error with only a modest increase to the computational time.

The rest of the paper is organized as follows: In Section 2 we outline the problem under consideration, the linearized Euler equations in 2-D with no advection, solved in an infinite domain with NRBCs on all four sides. Section 3 details the NRBCs and their application to the linearized Euler equations. In Section 4 we derive the Klein-Gordon equation from the linearized Euler equations with no mean flow. We discuss the finite difference discretization for the NRBCs and the interior scheme in Sections 5 and 6 , and we provide a numerical example in Section 7 . We then list some areas for further research (Section 8) and summarize our results (Section 9).

\section{Problem Statement}

Consider the linearized Euler equations in an open domain. For simplicity we assume that the domain has a flat bottom and that there is no advection, although this assumption may be removed in future studies. A Cartesian coordinate system $(x, y)$ is introduced, as shown in Fig. 1.

The nonlinear Euler equations are

$$
\begin{aligned}
\partial_{t} \rho+\partial_{x}(\rho u)+\partial_{y}(\rho v) & =0 \\
\partial_{t} u+u \partial_{x} u+v \partial_{y} u+\frac{1}{\rho} \partial_{x} p & =f v \\
\partial_{t} v+u \partial_{x} v+v \partial_{y} v+\frac{1}{\rho} \partial_{y} p & =-f u \\
\partial_{t} p+u \partial_{x} p+v \partial_{y} p+\gamma p\left(\partial_{x} u+\partial_{y} v\right) & =0,
\end{aligned}
$$

where we use the following shorthand for partial derivatives

$$
\partial_{a}=\frac{\partial}{\partial a} \quad, \quad \partial_{a b}=\frac{\partial^{2}}{\partial a \partial b},
$$




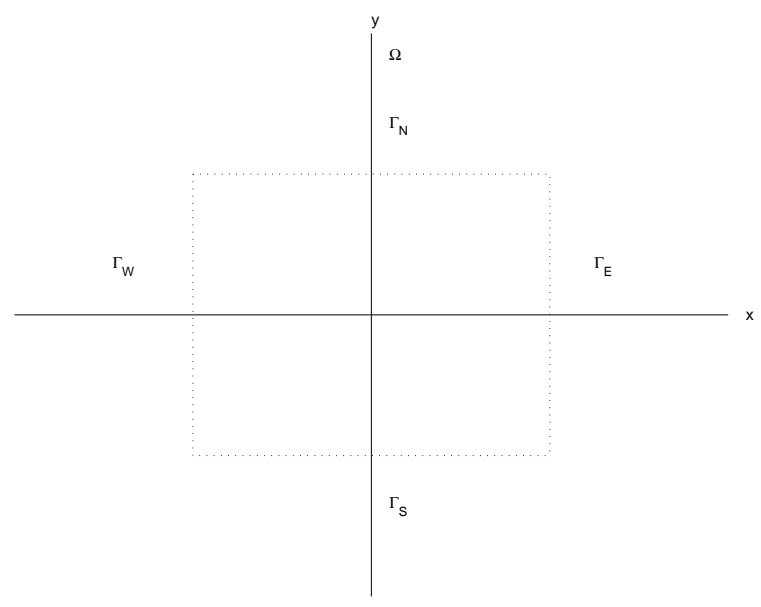

Figure 1: An open domain $\Omega$ truncated by artificial boundaries $\Gamma_{N}, \Gamma_{W}, \Gamma_{S}$, and $\Gamma_{E}$

and $t$ denotes the time, $u(x, y, t)$ and $v(x, y, t)$ the unknown velocities in the $x$ and $y$ directions, $\rho(x, y, t)$ the density, $p(x, y, t)$ the pressure, $f$ the constant Coriolis acceleration due to the Earth's rotation, and $\gamma=$ $c_{p} / c_{v}$ the constant ratio of specific heats. Linearizing these equations about mean zero velocities, constant mean density $\rho_{0}$ and constant mean pressure $p_{0}$ (see, e.g., [20] or [23]), we get:

$$
\begin{aligned}
\partial_{t} \rho+\rho_{0}\left(\partial_{x} u+\partial_{y} v\right) & =0 \\
\partial_{t} u+\frac{1}{\rho_{0}} \partial_{x} p & =f v \\
\partial_{t} v+\frac{1}{\rho_{0}} \partial_{y} p & =-f u \\
\partial_{t} p+\gamma p_{0}\left(\partial_{x} u+\partial_{y} v\right) & =0 .
\end{aligned}
$$

It can be shown that a single boundary condition must be imposed along the entire boundary to obtain a well-posed problem. At $\vec{x} \rightarrow \infty$ the solution is known to be bounded and not to include any incoming waves. To complete the statement of the problem, initial values for $u, v, p$ and $\rho$ are given at time $t=0$ in the entire domain.

We now truncate the infinite domain by introducing an artificial boundary $\Gamma$, with $\Gamma_{N}$ located at $y=y_{N}, \Gamma_{W}$ located at $x=x_{W}, \Gamma_{S}$ located at $y=y_{S}$, and $\Gamma_{E}$ located at $x=x_{E}$ (see dotted lines in Figure 1). To obtain a well-posed problem in the finite domain $\Omega$ we need, instead of the condition at infinity, a single boundary condition on each of the artificial boundaries $\Gamma_{N, W, S, E}$. This should be a Non-Reflecting Boundary Condition (NRBC). We shall apply a high-order NRBC for the variables, as described in the following section. 


\section{Higdon-type NRBCs}

On the artificial boundaries $\Gamma$ we use one of the Higdon NRBCs [19]. These NRBCs were presented and analyzed in a sequence of papers [13], [15]-[18] for non-dispersive acoustic and elastic waves, and were extended in [19] for dispersive waves. Their main advantages are as follows:

1. The Higdon NRBCs are very general, namely they apply to a variety of wave problems, in one, two, and three dimensions and in various configurations.

2. They form a sequence of NRBCs of increasing order. This enables one, in principle (leaving implementational issues aside for the moment), to obtain solutions with unlimited accuracy.

3. The Higdon NRBCs can be used, without any difficulty, for dispersive wave problems and for problems in stratified media. Most other available NRBCs are either designed for non-dispersive media (as in acoustics and electromagnetics) or are of low order (as in meteorology and oceanography).

The scheme used here is different than the original Higdon scheme [19] in the following ways:

1. The discrete Higdon conditions were developed in the literature up to third order only, because of their algebraic complexity which increases rapidly with the order. Givoli and Neta [8] showed how to easily implement these conditions to an arbitrarily high order. The scheme is coded once and for all for any order; the order of the scheme is simply an input parameter.

2. The original Higdon conditions were applied to the Klein-Gordon linear wave equation and to the elastic equations. Here we show how to apply them to the linearized Euler equations (2).

3. The Higdon NRBCs involve some parameters which must be chosen. Higdon [19] discusses some general guidelines for their manual a priori choice by the user. Neta et. al. [25] showed how a simple choice for these parameters can dramatically simplify the calculations and enable implementation of NRBCs of much higher order with less computational overhead.

The Higdon NRBC of order $J$ is

$$
H_{J}: \quad\left[\prod_{j=1}^{J}\left(\partial_{t}+C_{j} \partial_{x}\right)\right] \eta=0 \text { on } \Gamma_{E},
$$

where $\eta$ represents any one of the state variables $\rho, u, v, p$. Here, the $C_{j}$ are parameters which have to be chosen and which signify phase speeds in the $x$-direction. The boundary condition (3) is exact for all waves that propagate with an $x$-direction phase speed equal to any of $C_{1} \ldots C_{J}$. This is easy to see from the reflection coefficient (see below). For the boundary $\Gamma_{W}$ we replace $\partial_{x}$ by $-\partial_{x}$. Likewise, on $\Gamma_{N, S}$ we use $\pm \partial_{y}$. Givoli and Neta [8] and Dea et. al. [2] summarize several observations about these NRBCs, most of which we omit here for brevity. One point is worth 
repeating: For the Klein-Gordon equation, Higdon showed [19] that the reflection coefficient for an $\mathrm{NRBC}$ of order $J$ can be given by:

$$
|R|=\prod_{j=1}^{J}\left|\frac{C_{j}-C_{x}}{C_{j}+C_{x}}\right|,
$$

where $C_{x}$ is the wave speed in the $x$ direction. Note that this reflection coefficient is a product of $J$ factors, each of which is less than one. Consequently, increasing the order $J$ of the NRBC will result in reduced reflections, even if the $C_{j}$ values are suboptimal. We will take advantage of this fact when we discuss the NRBC discretization in Sec. 5, where we show that a certain choice of $C_{j}$ can reduce the computational complexity from $O\left(3^{J}\right)$ to $O\left(J^{2}\right)$.

\section{Equivalence of Linearized Euler Equa- tions and Klein-Gordon Equation}

Higdon showed in [19] that this NRBC formulation is compatible with the Klein-Gordon (dispersive wave) equation

$$
\partial_{t}^{2} \eta-C_{0}^{2} \nabla^{2} \eta+f^{2} \eta=0
$$

Hence, if we can show that (2) is equivalent to (5), we can claim that this NRBC formulation will be stable here.

Differentiate (2d) with respect to $t$

$$
\partial_{t t} p+\gamma p_{0}\left(\partial_{x t} u+\partial_{y t} v\right)=0
$$

Now differentiate $(2 \mathrm{~b})$ with respect to $x$ and (2c) with respect to $y$ and add

$$
\partial_{x t} u+\partial_{t y} v+\frac{1}{\rho_{0}}\left(\partial_{x x} p+\partial_{y y} p\right)=f\left(\partial_{x} v-\partial_{y} u\right) .
$$

Now substitute (7) into (6)

$$
\partial_{t t} p-\frac{\gamma p_{0}}{\rho_{0}}\left(\partial_{x x} p+\partial_{y y} p\right)+f \gamma p_{0}\left(\partial_{x} v-\partial_{y} u\right)=0 .
$$

Differentiate (2b) with respect to $y$ and (2c) with respect to $x$ and subtract

$$
\partial_{y t} u-\partial_{x t} v=f\left(\partial_{y} v+\partial_{x} u\right) .
$$

Combine terms to get

$$
\partial_{x} u+\partial_{y} v=-\frac{1}{f} \partial_{t}\left(\partial_{x} v-\partial_{y} u\right)
$$

Combine (2d) and (10) to get

$$
\partial_{t} p=\frac{\gamma p_{0}}{f} \partial_{t}\left(\partial_{x} v-\partial_{y} u\right)
$$

Integrate (11) with respect to time to get

$$
f\left(p-p_{0}\right)=\gamma p_{0}\left(\partial_{x} v-\partial_{y} u\right) .
$$


Finally, substitute (12) into (8)

$$
\partial_{t t} p-\frac{\gamma p_{0}}{\rho_{0}} \nabla^{2} p+f^{2}\left(p-p_{0}\right)=0
$$

which gives us the Klein-Gordon equation for the pressure perturbation $p-p_{0}$ with wave speed $\sqrt{\gamma p_{0} / \rho_{0}}$.

\section{Discretization of NRBCs}

The Higdon condition $H_{J}$ is a product of $J$ operators of the form $\partial_{t}+C_{j} \partial_{x}$. Consider the following finite difference approximations (see e.g. [27]):

$$
\partial_{t} \simeq \frac{I-S_{t}^{-}}{\delta t}, \partial_{x} \simeq \frac{I-S_{x}^{-}}{\delta x} .
$$

In (14), $\delta t$ and $\delta x$ are, respectively, the time-step size and grid spacing in the $x$ direction, $I$ is the identity operator, and $S_{t}^{-}$and $S_{x}^{-}$are backward shift operators defined by

$$
S_{t}^{-} \eta_{p q}^{n}=\eta_{p q}^{n-1}, S_{x}^{-} \eta_{p q}^{n}=\eta_{p-1, q}^{n} .
$$

Here and elsewhere, $\eta_{p q}^{n}$ is the FD approximation of $\eta(x, y, t)$ at grid point $\left(x_{p}, y_{q}\right)$ and at time $t_{n}$. We use (14) in (3) to obtain:

$$
\left[\prod_{j=1}^{J}\left(\frac{I-S_{t}^{-}}{\delta t}+C_{j} \frac{I-S_{x}^{-}}{\delta x}\right)\right] \eta_{E q}^{n}=0 .
$$

Here, the index $E$ corresponds to a grid point on the boundary $\Gamma_{E}$. On the other open boundaries, the normal derivatives and shift operators should be adjusted accordingly.

Givoli and Neta [8] showed how to implement the Higdon NRBCs to any order using a simple algorithm. Their algorithm requires the summation of $O\left(3^{J}\right)$ terms. However, if we make the simplification

$$
C_{j} \equiv C_{0} \forall j \in 1 \ldots J
$$

then we can simplify the summation to

$$
\begin{aligned}
Z & \equiv\left(a I+b S_{t}^{-}+c S_{x}^{-}\right)^{J} \eta_{E q}^{n} \\
& =\sum_{\beta=0}^{J} \sum_{\gamma=0}^{J-\beta} \frac{J !}{\alpha ! \beta ! \gamma !} a^{\alpha} b^{\beta} c^{\gamma} S_{t}^{-\beta} S_{x}^{-\gamma} \eta_{E q}^{n}=0 \\
\text { where } a & =1-c \\
b & =-1 \\
c & =-C_{0} \frac{\delta t}{\delta x} \\
\alpha & =J-\beta-\gamma .
\end{aligned}
$$

This summation consists of only $O\left(J^{2}\right)$ terms, reducing the computational time considerably. As shown in the discussion of the reflection coefficient in Sec. 3, this choice of $C_{j}$ will be less accurate than an optimized selection 
of $C_{j}$ 's based on dispersive wave speeds; however, the results will still improve as we increase our order $J$. We accept the slight loss of accuracy in exchange for the significant increase in speed. Note also that the $J=1$ case, with this choice of $C_{j}$, is the classic Sommerfeld radiation condition. We choose this particular NRBC wave speed as a middle ground between the phase speeds of the dispersive waves and the lowered normal velocities associated with non-zero incidence angles.

\section{Discretization in the Interior}

We consider explicit FD interior discretization schemes for the linearized Euler equations (2) to be used in conjunction with the $H_{J}$ condition. The interaction between the $H_{J}$ condition and the interior scheme is a source of concern, since simple choices for an explicit interior scheme turn out to give rise to instabilities. The effort to contrive a compatible discretization scheme was described in [2] for the linearized Euler equations without Coriolis. There, we used a one-sided differencing scheme for the interior, such that the discretized system was equivalent to the standard secondorder centered-difference scheme for the scalar wave equation in $p$, which Higdon proved in [19] was compatible with the NRBC formulation. However, subsequent work has shown that adding the Coriolis terms to this scheme results in a system which cannot be converted to the Klein-Gordon equation. Hence, another approach is needed.

Let us reconsider a second-order centered-difference (leap-frog) scheme,

$$
\eta^{\prime}(a) \approx \frac{\eta(a+\delta a)-\eta(a-\delta a)}{2 \delta a} .
$$

where $\eta$ denotes any of our four state variables, and $a$ denotes any of our spatial or temporal variables. Using the shift operator notation from the preceding section, we define our difference approximations as

$$
\begin{aligned}
\Delta_{a} & =\frac{S_{a}^{+}-S_{a}^{-}}{2 \delta a} \\
a & \in\{x, y, t\} .
\end{aligned}
$$

From this definition, we propose the following discretization scheme for (2):

$$
\begin{aligned}
\Delta_{t} \rho+\rho_{0}\left(\Delta_{x} u+\Delta_{y} v\right) & =0 \\
\Delta_{t} u+\frac{1}{\rho_{0}} \Delta_{x} p & =f v \\
\Delta_{t} v+\frac{1}{\rho_{0}} \Delta_{y} p & =-f u \\
\Delta_{t} p+\gamma p_{0}\left(\Delta_{x} u+\Delta_{y} v\right) & =0 .
\end{aligned}
$$

Apply $\Delta_{x}$ to $(21 \mathrm{~b}), \Delta_{y}$ to $(21 \mathrm{c}), \Delta_{t}$ to $(21 \mathrm{~d})$, and make the appropriate substitution. This gives us

$$
\Delta_{t} \Delta_{t} p=\frac{\gamma p_{0}}{\rho_{0}}\left(\Delta_{x} \Delta_{x} p+\Delta_{y} \Delta_{y} p\right)-f \gamma p_{0}\left(\Delta_{x} v-\Delta_{y} u\right)
$$


If $f=0$, then this discretization is equivalent to a scalar wave discretization. Hence, in the absence of Coriolis forces, the discretization scheme (21) is compatible with the discrete Higdon NRBCs (as modified below).

Continuing our derivation, we apply $\Delta_{y}$ to $(21 \mathrm{~b})$ and $\Delta_{x}$ to (21c), then subtract and combine terms to get

$$
\Delta_{t}\left(\Delta_{y} u-\Delta_{x} v\right)=f\left(\Delta_{y} v+\Delta_{x} u\right) .
$$

We then substitute (21d) into this result to get

$$
\Delta_{t}\left(\Delta_{y} u-\Delta_{x} v\right)=-\frac{f}{\gamma p_{0}} \Delta_{p}
$$

If we apply $\Delta_{t}$ to $(22)$ and incorporate $(24)$, we get

$$
\Delta_{t}\left[\Delta_{t} \Delta_{t} p-\frac{\gamma p_{0}}{\rho_{0}}\left(\Delta_{x} \Delta_{x} p+\Delta_{y} \Delta_{y} p\right)+f^{2} p\right]=0 .
$$

Thus, the quantity inside the brackets is constant from one time step to the next. Since this equation applies to our initial state, then the quantity within the brackets must initially be zero and thus remain zero always; hence,

$$
\Delta_{t} \Delta_{t} p=\frac{\gamma p_{0}}{\rho_{0}}\left(\Delta_{x} \Delta_{x} p+\Delta_{y} \Delta_{y} p\right)-f^{2} p .
$$

If we expand our $\Delta_{[x, y, t]}$ symbols into their corresponding shift operators and apply them to the state variable $p$, we see that $(26)$ is actually

$$
\begin{aligned}
\frac{p_{i, j}^{n+2}-2 p_{i, j}^{n}+p_{i, j}^{n+2}}{(2 \delta t)^{2}}= & \frac{\gamma p_{0}}{\rho_{0}}\left(\frac{p_{i+2, j}^{n}-2 p_{i, j}^{n}+p_{i-2, j}^{n}}{(2 \delta x)^{2}}+\right. \\
& \left.\frac{p_{i, j+2}^{n}-2 p_{i, j}^{n}+p_{i, j-2}^{n}}{(2 \delta y)^{2}}\right)-f^{2} p_{i, j}^{n}
\end{aligned}
$$

This equation is the standard second-order centered-difference scheme for the Klein-Gordon equation on a double-sized grid. Hence, the appropriate discretization for the Higdon scheme is not (14) but

$$
\partial_{t} \simeq \frac{I-S_{t}^{-2}}{2 \delta t}, \partial_{x} \simeq \frac{I-S_{x}^{-2}}{2 \delta x} .
$$

\section{Numerical Example}

Let us consider a simple numerical example. We look at a square domain $10 \mathrm{~km}$ on each side, subdividing it into a $100 \times 100$ computational domain with the Higdon-like NRBCs on all four sides (see Fig. 1). We define the NRBC for all four state variables, because we use a centered-difference discretization. A one-sided discretization scheme would enable us to use a natural boundary condition on one side or another, but previous experiments have shown that such a scheme is unstable.

Using a mean atmoshperic density of $1.2 \frac{\mathrm{kg}}{\mathrm{m}^{3}}$ and pressure of $1.01 \times$ $10^{5} \frac{\mathrm{N}}{\mathrm{m}^{2}}$ [12], a Coriolis value of $f=7.292116 \times 10^{-5} \frac{\mathrm{rad}}{\mathrm{s}}$ [28], and zero 
advection, our initial condition is a cosine bubble in the center of the domain:

$$
\begin{aligned}
& p_{x, y}^{0}=\left\{\begin{aligned}
p_{0}\left(1+\frac{\cos \left(\frac{\pi}{2} \frac{d}{r}\right)}{100}\right) & : d \leq r \\
p_{0} & : \text { otherwise }
\end{aligned}\right. \\
& \rho_{x, y}^{0}=\left\{\begin{aligned}
\rho_{0}\left(\frac{p_{x, y}^{0}}{p_{0}}\right)^{\frac{c_{v}}{c_{p}}}: & d \leq r \\
\rho_{0}: & \text { otherwise }
\end{aligned}\right.
\end{aligned}
$$

where

$$
\begin{aligned}
d & =\sqrt{\left(x-x_{C}\right)^{2}+\left(y-y_{C}\right)^{2}} \\
r & =1 \mathrm{~km},
\end{aligned}
$$

and $x_{C}$ and $y_{C}$ denote the center of the domain. The initial condition for $\rho$ is chosen to maintain constant potential temperature with the pressure perturbation [3]. For comparison, our reference solution domain is $30 \mathrm{~km}$ wide and $30 \mathrm{~km}$ high, with the domain of interest in the center. We define the normalized error norm for each state variable $\eta$ as

$$
E_{\eta}=\frac{\sqrt{\sum_{i=1}^{N_{x}} \sum_{j=1}^{N_{y}}\left(\eta_{J}(i, j)-\eta_{0}(i, j)\right)^{2}}}{\sqrt{\sum_{i=1}^{N_{x}} \sum_{j=1}^{N_{y}} \eta_{0}(i, j)^{2}}},
$$

where $N_{x}, N_{y}$ are the number of grid points in the $x$ and $y$ directions, respectively, $\eta_{J}$ is a solution state variable using the $J$-order NRBC, and $\eta_{0}$ is the reference solution. We divide by the norm of $\eta_{0}$ so that the error norms of each state variable are approximately the same order of magnitude. Our time step is set to $90 \%$ of the maximum $\delta t$ allowed by the CFL limit, where the CFL limit is

$$
\left(C_{0} \frac{\delta t}{\delta x}\right)^{2}+\left(C_{0} \frac{\delta t}{\delta y}\right)^{2} \leq 1,
$$

where $C_{0}=\sqrt{\gamma p_{0} / \rho_{0}}$ is the acoustic wave speed. Using the discretization scheme (21), we run the simulation up to $t=24$, long enough for the primary wave to exit the computational domain with the wave trough just passing through the corners. Figs. $2-5$ show the four state variables at the end of the run for $J=10$. Fig. 6 compares the state variable $u$ at the end of the $J=1$ and $J=10$ cases; note the dramatic reduction in spurious reflection in the $J=10$ case. Table 1 and Fig. 7 shows the error norms (30) for each state variable as $J$ goes from 1 to 10.

One note about the time step: We have found experimentally that if $\delta t$ is set to exactly the CFL limit, then the error norm for $J=10$ is only approximately $55 \%$ the $J=1$ error norm. On the other hand, if we use exactly half the CFL limit for our $\delta t$, then the error norm reduction is on the order of $99.5 \%$. We chose $90 \%$ of the maximum $\delta t$ as a compromise between increased accuracy and increased time step size. 


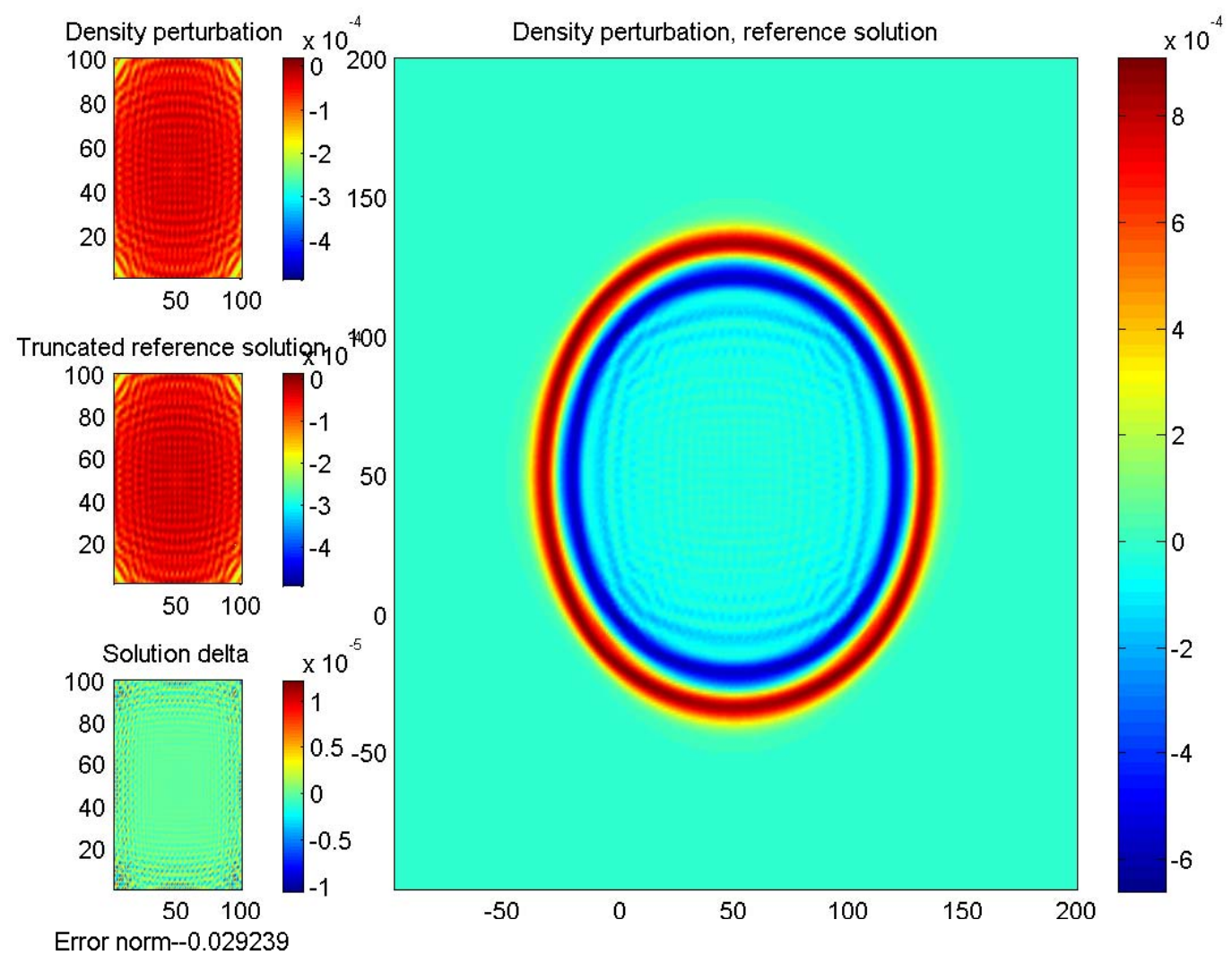

Figure 2: The solution for the density $\rho$ using $J=10$. (TL) Computed solution using NRBCs. (Right) Reference solution. (CL) Reference solution domain corresponding to NRBC solution domain. (BL) Delta between computed and reference solutions, with error norm computed by (30).

\section{Areas for Further Research}

The preceding example demonstrates, in a limited setting, that high-order Higdon NRBCs are compatible with the linearized Euler equations. However, there are far more areas to explore in this implementation. The following list shows some of the areas available for future research, some of which are currently under investigation by the authors:

1. Thorough investigation of the long-time stability for large $J$.

2. Extending the scheme to the case of the linearized Euler equations with a nonzero mean flow (advection).

3. Extending the scheme to include the effects of gravity (in the $x z$ plane). 


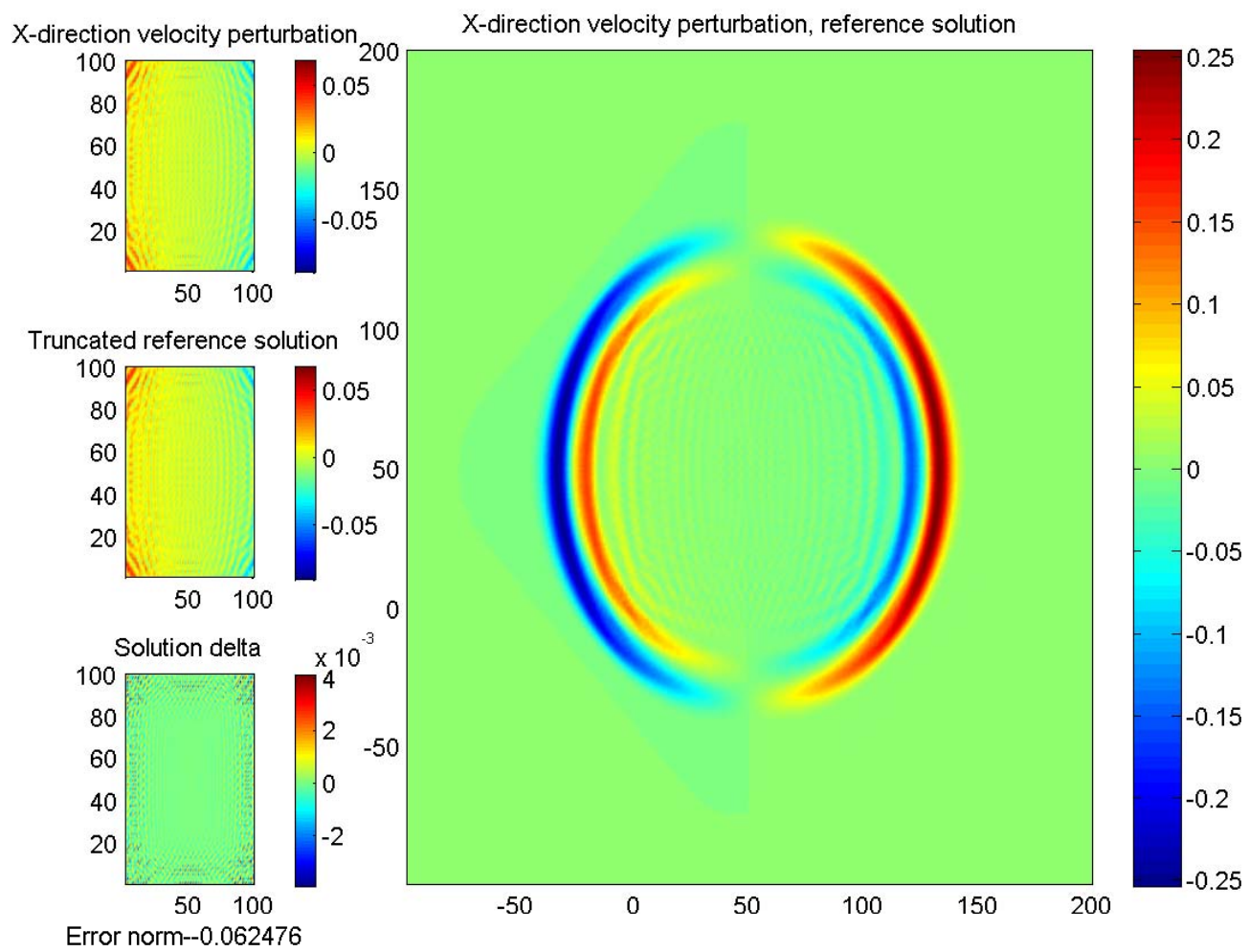

Figure 3: The solution for the $x$-direction velocity $u$ using $J=10$. (TL) Computed solution using NRBCs. (Right) Reference solution. (CL) Reference solution domain corresponding to NRBC solution domain. (BL) Delta between computed and reference solutions, with error norm computed by (30).

4. Implementing the scheme with auxiliary variables, using finite differences and finite elements, using both the Givoli-Neta AV formulation [10] and the Hagstrom-Warburton variation [11].

5. Extending the scheme to permit incoming waves, for example, in a nested mesoscale model.

6. Experimenting with the use of the NRBC with the nonlinear Euler equations (1) in the computational domain. (Need to find a stable interior scheme-NRBC combination.).

\section{Conclusion}

In this paper, we have shown that Higdon-type NRBCs are compatible with the linearized Euler equations with Coriolis and zero mean flow. These NRBCs provide greater accuracy (reduced spurious reflection) than 


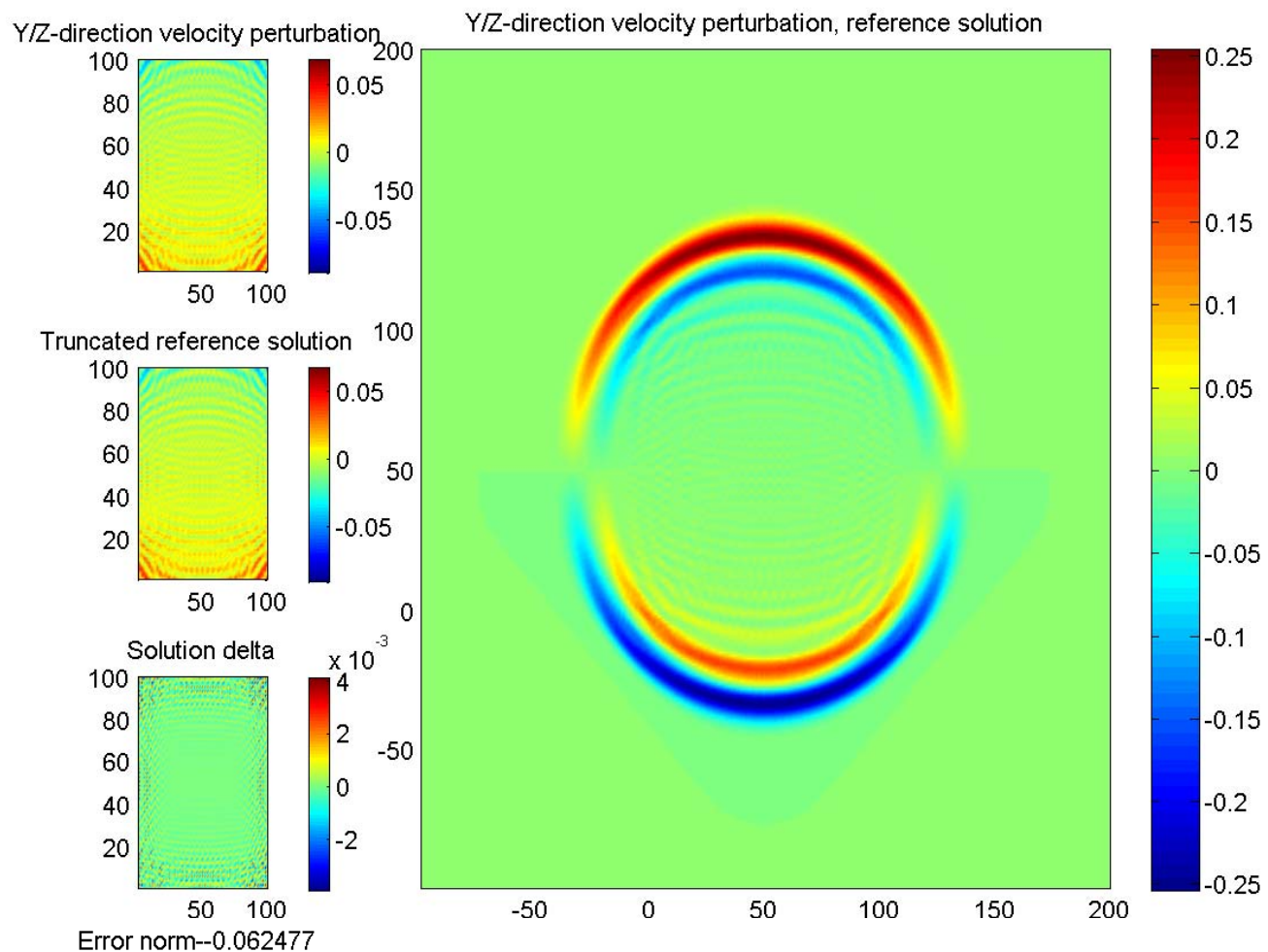

Figure 4: The solution for the $y$-direction velocity $v$ using $J=10$. (TL) Computed solution using NRBCs. (Right) Reference solution. (CL) Reference solution domain corresponding to NRBC solution domain. (BL) Delta between computed and reference solutions, with error norm computed by (30).

the basic Sommerfeld boundary condition. A prototypical implementation was developed, and a numerical example demonstrating the capabilities of the scheme was provided.

\section{Acknowledgments}

The authors would like to express their appreciation to the Naval Postgraduate School for its support of this research. The first author is also indebted to the Air Force Institute of Technology for its support.

\section{References}

[1] Bérenger, J., "A Perfectly Matched Layer for the Absorption of Electromagnetic Waves," Journal of Computational Physics 114, pp.185- 


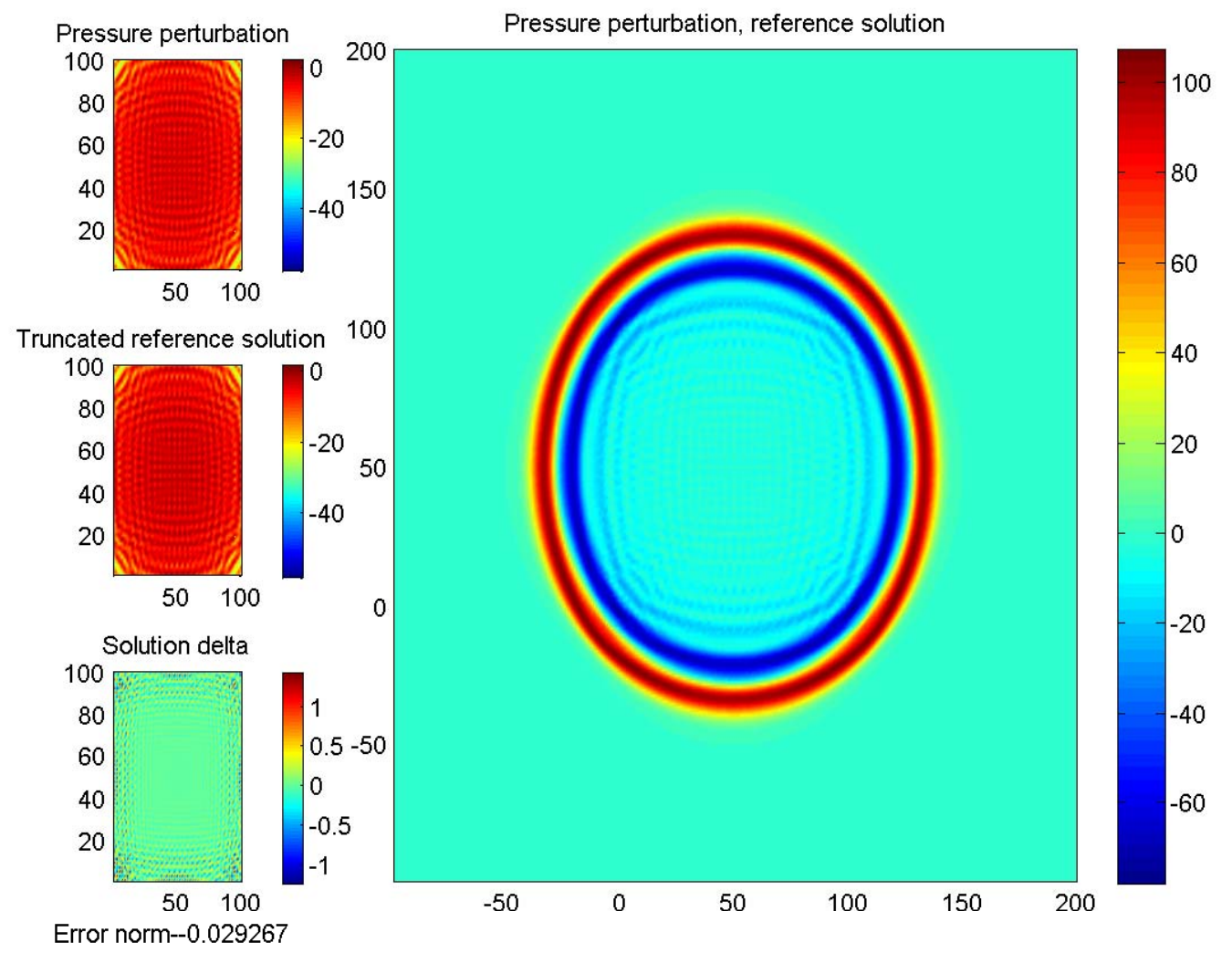

Figure 5: The solution for the pressure $p$ using $J=10$. (TL) Computed solution using NRBCs. (Right) Reference solution. (CL) Reference solution domain corresponding to NRBC solution domain. (BL) Delta between computed and reference solutions, with error norm computed by (30).

200, 1994.

[2] Dea, J., F. Giraldo, and B. Neta, High-Order Higdon Non-Reflecting Boundary Conditions for the Linearized Euler Equations, NPS-MA07-001, Naval Postgraduate School, Monterey, CA, 2007.

[3] Durran, D., Numerical Methods for Wave Equations in Geophysical Fluid Dynamics, Springer, New York, 1999.

[4] Engquist, B., and A. Majda, "Absorbing Boundary Conditions for the Numerical Simulation of Waves," Mathematics of Computation 31, pp.629-651, 1977.

[5] Engquist, B., and A. Majda, "Radiation Boundary Conditions for Acoustic and Elastic Wave Calculations," Communications on Pure and Applied Mathematics 32, pp.313-357, 1979. 

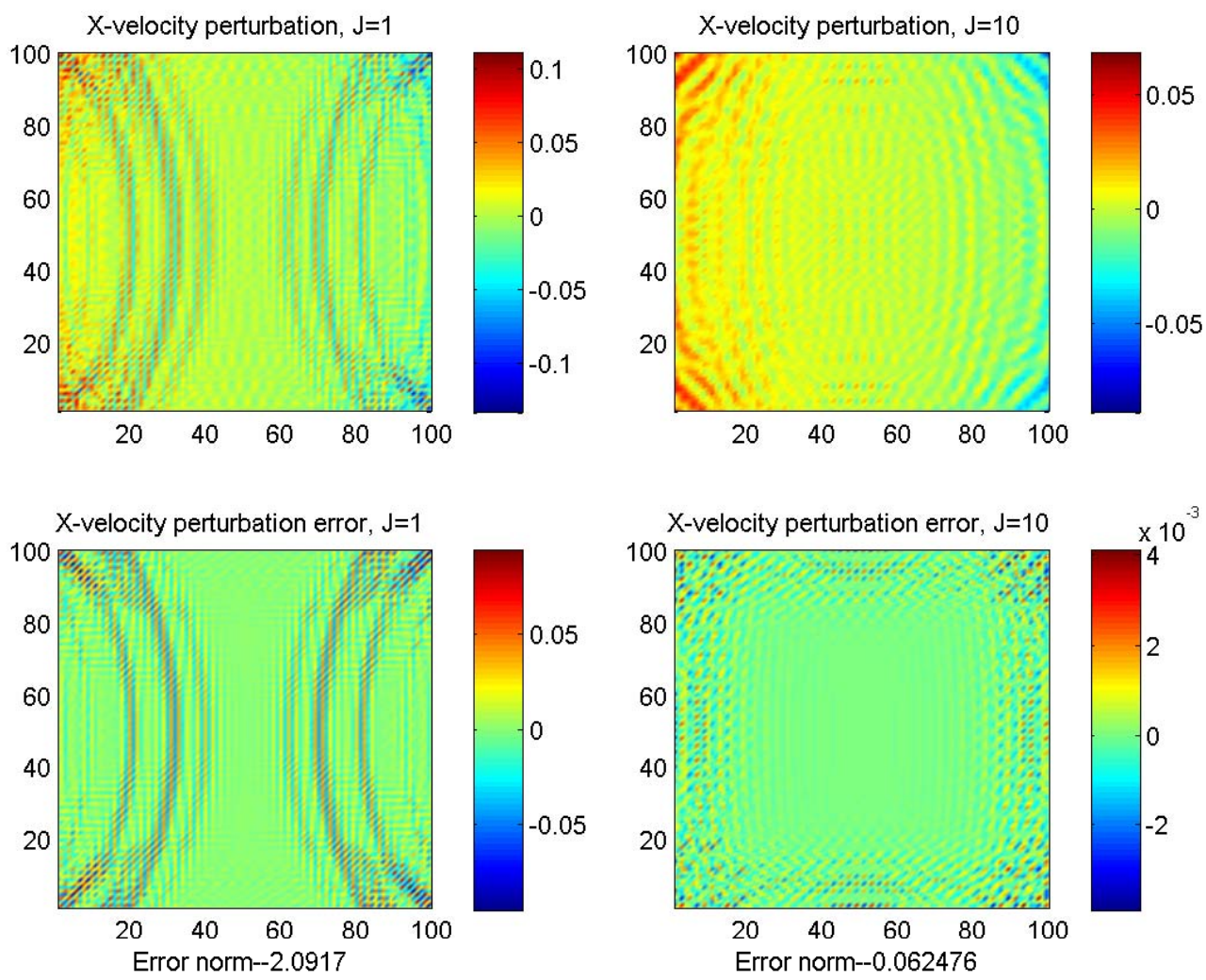

Figure 6: A comparison of solutions for $u$ using $J=1$ and $J=10$. (TL) Computed solution using $J=1$. (TR) Computed solution using $J=10$. (BL) Delta between reference solution and $J=1$ solution, with error norm computed by (30). (BR) Delta between reference solution and $J=10$ solution, with error norm computed by (30).

[6] Giraldo, F., and M. Restelli, "A Study of Spectral Element and Discontinuous Galerkin Methods for Mesoscale Atmospheric Modeling: Equation Sets and Test Cases," submitted to Journal of Computational Physics, 2007.

[7] Givoli, D., and B. Neta, High-Order Higdon Non-Reflecting Boundary Conditions for the Shallow Water Equations, NPS-MA-02-001, Naval Postgraduate School, Monterey, CA, 2002.

[8] Givoli, D., and B. Neta, "High-Order Non-Reflecting Boundary Conditions for Dispersive Waves," Wave Motion 37, pp.257-271, 2003.

[9] Givoli, D., and B. Neta, "High-Order Non-Reflecting Boundary Conditions for the Dispersive Shallow Water Equations, Journal of Computational and Applied Mathematics 158, pp.49-60, 2003. 

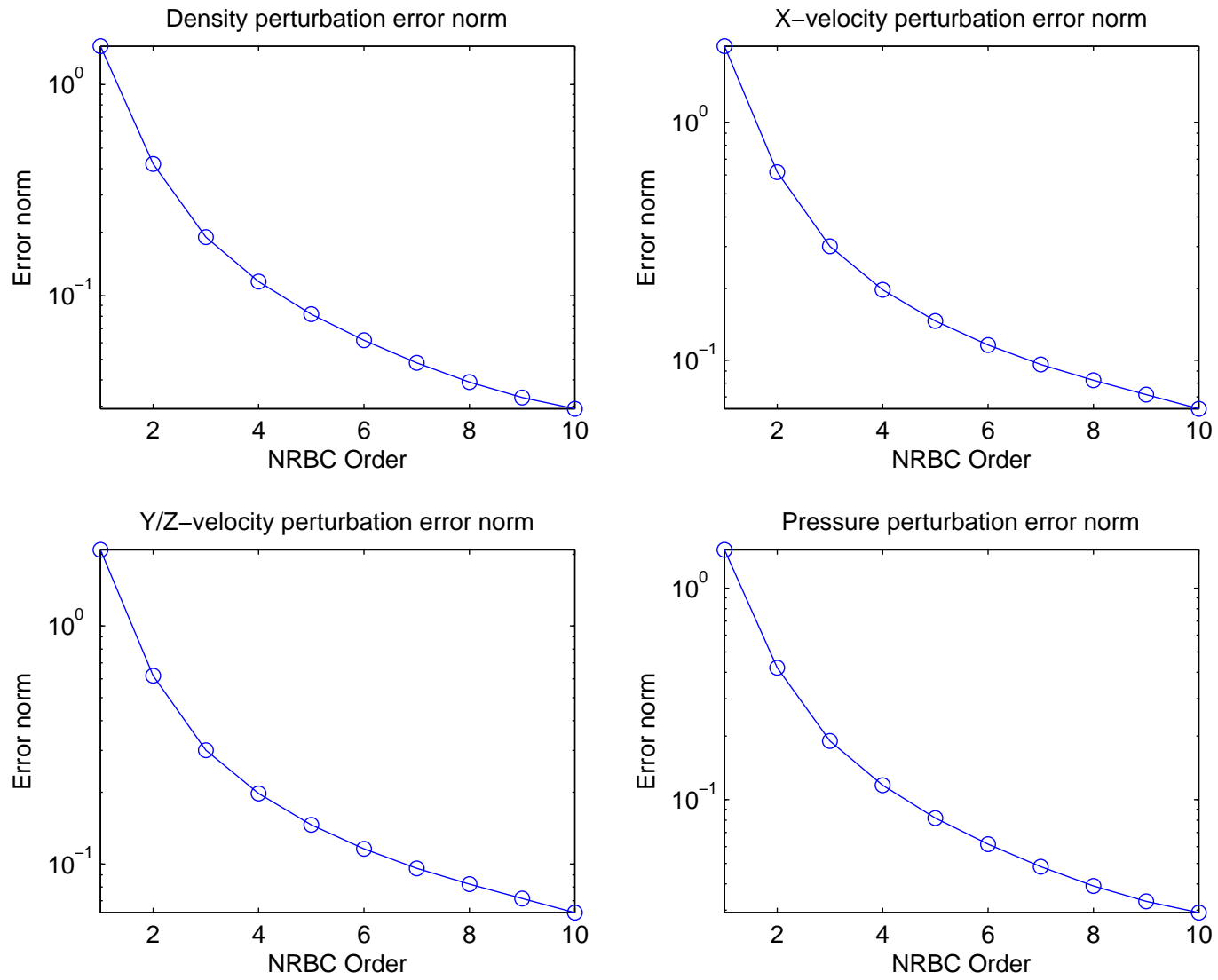

Figure 7: Logarithmic plot of state variable error norms (30) for $J \in 1 \ldots 10$ with discretization scheme (21). (TL) Error norms for $\rho$. (TR) Error norms for $u$. (BL) Error norms for $v$. (BR) Error norms for $p$. 


\begin{tabular}{r|l|l|l|l}
$J$ & $E_{\rho}$ & $E_{u}$ & $E_{v}$ & $E_{p}$ \\
\hline 1 & 1.5191 & 2.0917 & 2.0917 & 1.5205 \\
2 & 0.42052 & 0.61777 & 0.61777 & 0.42092 \\
3 & 0.18953 & 0.30055 & 0.30054 & 0.18971 \\
4 & 0.11677 & 0.19766 & 0.19766 & 0.11689 \\
5 & 0.081815 & 0.14588 & 0.14588 & 0.081893 \\
6 & 0.061569 & 0.11564 & 0.11564 & 0.061628 \\
7 & 0.048183 & 0.095798 & 0.095797 & 0.04823 \\
8 & 0.03908 & 0.082285 & 0.082284 & 0.039118 \\
9 & 0.033036 & 0.071617 & 0.071617 & 0.033067 \\
10 & 0.029239 & 0.062476 & 0.062477 & 0.029267
\end{tabular}

Table 1: Error norms (30) for $J \in 1 \ldots 10$ with discretization scheme (21)

[10] Givoli, D., and B. Neta, "High-Order Non-Reflecting Boundary Scheme for Time-Dependent Waves," Journal of Computational Physics 186, pp.24-26, 2003.

[11] Hagstrom, T., and T. Warburton, "A New Auxiliary Variable Formulation of High-Order Local Radiation Boundary Conditions: Corner Compatibility Conditions and Extension to First-Order Systems," Wave Motion 39, pp.327-338, 2004.

[12] Halliday, D., and R. Resnick, Fundamentals of Physics, Third Edition Extended, John Wiley and Sons, New York, 1988.

[13] Higdon, R., "Absorbing Boundary Conditions for Difference Approximations to the Multi-Dimensional Wave Equation," Mathematics of Computation 47, pp.437-459, 1986.

[14] Higdon, R., "Initial-Boundary Value Problems for Linear Hyperbolic Systems," SIAM Review 28, pp.177-217, 1986.

[15] Higdon, R., "Numerical Absorbing Boundary Conditions for the Wave Equation," Mathematics of Computation 49, pp.65-90, 1987.

[16] Higdon, R., "Radiation Boundary Conditions for Elastic Wave Propagation," SIAM Journal on Numerical Analysis 27, pp.831-869, 1990.

[17] Higdon, R., "Absorbing Boundary Conditions for Elastic Waves," Geophysics 56, pp.231-241, 1991.

[18] Higdon, R., "Absorbing Boundary Conditions for Acoustic and Elastic Waves in Stratified Media," Journal of Computational Physics 101, pp.386-418, 1992.

[19] Higdon, R., "Radiation Boundary Conditions for Dispersive Waves," SIAM Journal on Numerical Analysis 31, pp.64-100, 1994.

[20] Hu, F., "On Absorbing Boundary Conditions for Linearized Euler Equations by a Perfectly Matched Layer," Journal of Computational Physics 129, pp.201-219, 1996.

[21] Hu, F., "A Stable, Perfectly Matched Layer for Linearized Euler Equations in Unsplit Physical Variables," Journal of Computational Physics 173, pp.455-480, 2001. 
[22] Hu, F., "A Perfectly Matched Layer Absorbing Boundary Condition for Linearized Euler Equations with a Non-Uniform Mean Flow," Journal of Computational Physics 208, pp.469-492, 2005.

[23] Kröner, D., "Absorbing Boundary Conditions for the Linearized Euler Equations in 2-D," Mathematics of Computation 57, pp.153167, 1991.

[24] Navon, I., B. Neta, and M. Hussaini, "A Perfectly Matched Layer Approach to the Linearized Shallow Water Equations Models," Monthly Weather Review 132, pp.1369-1378, 2004.

[25] Neta, B., V. van Joolen, J. Dea, and D. Givoli, "Application of High-Order Higdon Non-Reflecting Boundary Conditions to Linear Shallow Water Models," to appear in Communications in Numerical Methods in Engineering, published online at www.interscience.wiley.com, DOI: 10.1002/cnm.1044, 2007.

[26] Orlanski, I., "A Simple Boundary Condition for Unbounded Hyperbolic Flows," Journal of Computational Physics 21, pp.251-269, 1976.

[27] Tannehihll, J., D. Anderson, and R. Pletcher, Computational Fluid Mechanics and Heat Transfer, Second Edition, Taylor \& Francis, Washington, DC, 1997.

[28] Vallado, D., Fundamentals of Astrodynamics and Applications, Second Edition, Microcosm Press, El Segundo, CA, 2001.

[29] Van Joolen, V., Application of Higdon Non-Reflecting Boundary Conditions to Shallow Water Models, PhD Dissertation, Naval Postgraduate School, Monterey, CA, 2003.

[30] Van Joolen, V., D. Givoli, and B. Neta, "High-Order Non-Reflecting Boundary Conditions for Dispersive Waves in Cartesian, Cylindrical and Spherical Coordinate Systems," International Journal of Computational Fluid Dynamics 17, pp.263-274, 2003.

[31] Van Joolen, V., B. Neta, and D. Givoli, "A Stratified Dispersive Wave Model with High-Order Non-Reflecting Boundary Conditions," Computers and Mathematics with Applications 48, pp.1167-1180, 2004.

[32] Van Joolen, V., B. Neta, and D. Givoli, "High-Order Higdon-Like Boundary Conditions for Exterior Transient Wave Problems," International Journal for Numerical Methods in Engineering 63, pp.10411068, 2005. 